Voix et Images

voixetimages

\title{
Le monde, comme un théâtre
}

\section{LUCIE ROBERT}

Volume 42, numéro 3 (126), printemps-été 2017

URI : https://id.erudit.org/iderudit/1041055ar

DOI : https://doi.org/10.7202/1041055ar

Aller au sommaire du numéro

Éditeur(s)

Université du Québec à Montréal

\section{ISSN}

0318-9201 (imprimé)

1705-933X (numérique)

Découvrir la revue

Citer ce compte rendu

ROBERT, L. (2017). Compte rendu de [Le monde, comme un théâtre]. Voix et Images, 42(3), 147-153. https://doi.org/10.7202/1041055ar d'utilisation que vous pouvez consulter en ligne.

https://apropos.erudit.org/fr/usagers/politique-dutilisation/ 
D R A M A T U R G I E

Le monde, comme un théâtre

$++$

LUCIE ROBERT

Université du Québec à Montréal

All the world's a stage

And all the men and women merely players ${ }^{1}$.

Nombreux ont été les auteurs dramatiques qui ont représenté le monde comme un théâtre où chacun joue son rôle. Il y a là une typique conception de l'univers d'Ancien Régime, qui repose sur les apparences, mais aussi sur la croyance en une cosmogonie préétablie et déterminée par un dessein supérieur, et dont il faut conserver l'équilibre. Rester à sa place, se conduire comme il faut, voilà autant d'expressions qui nous viennent de cette époque et de cette conception. Toutefois, si les auteurs dramatiques ont pu peindre le monde ainsi, c'est que déjà s'était immiscé dans leur pensée un doute, l'idée d'une relativité à explorer plus avant, une tension singulière entre l'existence comme rôle figé et la vie réelle qui s'ouvre ou qui pourrait s'ouvrir sur un devenir non encore réalisé. Cette faille, qui met en tension deux plans de l'existence humaine, est explorée encore plus dans le roman à partir du XIX ${ }^{e}$ siècle. Car aucun romancier du siècle, de Mérimée à Zola, n'échappe à la fascination du thêâtre. Le roman du xixe siècle s'est construit contre le théâtre, sur l'échec du trompe-l'œil, au profit de la relation d'incertitude qu'engendre l'immixtion du temps et du personnage dans l'univers de l'action. Il n'en va pas autrement dans les œuvres contemporaines, et il faut croire que l'esthétique du thêâtre et l'esthétique du roman entretiennent toujours des rapports conflictuels.

En publiant ce qui fut son œuvre inaugurale, Le réel et le théâtral ${ }^{2}$, il y a un peu plus de quarante ans, Naïm Kattan poursuivait semblable réflexion, mais en la situant dans le passage entre deux civilisations, l'orientale et l'occidentale, qui entretiennent une relation distincte à la réalité. De son point de vue, l'Occident a médiatisé sa relation au religieux, au politique et au social en créant des modalités de

1 William Shakespeare, "As You Like It», The Unabridged William Shakespeare, édité par William George Clark et William Aldis Wright, Londres/Philadelphie, Running Press, p. 260. «Le monde entier est un thêâtre/Et tous, hommes et femmes, n'en sont que les acteurs» (traduction de François-Victor Hugo [Euvres complètes de William Shakespeare, t. VIII, Paris, Pagnerre libraire-éditeur, 1872, p. 327]).

2 Naïm Kattan, Le réel et le thêâtral, préface de Jean Grosjean, Montréal, Éditions HMH, coll. «Constantes», 1970, 188 p.; Paris, Denoël, coll. «Les lettres nouvelles», 1971, 181 p. 
représentations et d'artifices qui fondent de nombreux rituels ou cérémonials, fictions et fantasmes. Cette civilisation, que Kattan a découverte dans l'exil alors qu'il était étudiant à Paris, est en rupture avec le réel, et elle s'oppose au Bagdad de son enfance et de ses racines et, plus largement, à la culture orientale qu'il conçoit toujours comme favorisant le lien direct entre la personne et le réel. D'ailleurs, rappellet-il, il n'y a pas de thêâtre au Moyen-Orient, du moins pas de thêâtre comme nous le connaissons ici, et il n'y a pas de dramaturgie en langue arabe. Ce théâtral, découvert avec une certaine inquiétude quand il s'immisce au cœur des relations humaines, est paradoxalement une source de ravissement quand il devient une œuvre artistique qui explore les modalités de cette rupture. Car, toute sa carrière durant, Kattan est resté fidèle au thêâtre. Et voilà que vient de paraître l'intégrale de son œuvre dramatique, Thêâtre (1970-2014)33, qui regroupe dix-neuf textes écrits pour la scène ou pour la radio et dont plusieurs étaient, jusqu'à présent, demeurés inédits.

La présente publication est prise en charge par la collection «FrancoAmériques» sous la direction de Florence Davaille, collection qui «a pour vocation de publier des ouvrages sur les Amériques qui se disent ou se vivent en français» (8). Cet ouvrage est le premier de la collection et, à ce jour, encore le seul. Kattan a lui-même écrit une préface intitulée "La parole partagée», où il rappelle que, au thêâtre, «la personne qui prend la parole n'est pas seule» (17). L'écriture dramatique ouvre ainsi «l'univers de la réplique, du dialogue» (17), et elle ramène toute action à un échange de paroles entre deux ou plusieurs personnages. Entre le personnage et le spectateur, la parole, le dialogue transitent par le corps du comédien. De sorte que, redoublant le passage vécu par l'auteur entre les civilisations, de l'Orient vers l'Occident, «le théâtral exige un passage [et le] seul qui en est profondément et naturellement conscient est le comédien» (18). L'écriture dramatique, pour Kattan, représente donc une tentative de saisir et de conceptualiser l'essence même de cette civilisation, nouvelle pour lui, qu'il découvre au début de sa carrière d'écrivain, en même temps que cette nouvelle langue, le français, dont il apprivoise les possibles. Le réel et le théâtral, ce premier ouvrage dont il rappelle ici les grandes thèses, apparait comme une tentative pour faire état de cet itinéraire.

L'opposition entre le réel et le théâtral est aussi à l'origine de la première pièce publiée de Kattan: Les protagonistes ${ }^{4}$, qui met en scène précisément un groupe de comédiens aux prises avec le Mouvement Anti-Theâtral (le MAT), lequel entend éliminer l'hypocrisie des relations humaines en supprimant le théâtre. Devant la menace, qu'il faut bien qualifier de terroriste puisque certains comédiens ont déjã été assassinés au début de la pièce, les personnages sont réduits au silence: les thêâtres "sont fermés, les cinémas ne présentent plus que des bandes d'actualités et des documentaires» (471), la télévision ne diffuse que des nouvelles et un peu de musique. Les comédiens prennent le maquis et quelques-uns continuent de répéter leurs rôles, pour le jour où le théâtre reviendra. Peut-être découvriront-ils,

3 Naïm Kattan, Thêâtre (1970-2014), Mont-Saint-Aignan, Presses universitaires de Rouen et du Havre, coll. «Franco-Amériques», 2015, 533 p.

4 La pièce parut d'abord dans Écrits du Canada français, nº 35, 1972, p. 65-78, et a été reprise avec trois autres dans La discrétion. La neige. Le trajet. Les protagonistes, Montréal, Leméac, coll. «Thêâtre canadien», $1974,141 \mathrm{p}$. 
entre-temps, que l'essence du thêâtre n'est pas dans la production scénique achevée et «que ce qui compte vraiment, c'est de répéter» (495). Une autre pièce, Le cercle, explore semblable problématique en mettant en scène quelques-uns des dîners qui réunissent périodiquement un cercle d'amis chez Luc et Sylviane, personnages qui ont un rapport opposé à la réalité. Sylviane imagine des intrigues qui pourraient animer, déplacer, voire transformer les rapports qu'entretiennent ses hôtes et amis. Pour elle, la réalité ne se déploie que sur un mode hypothétique. Au contraire, Luc vit par procuration dans un réel qu'il voudrait stable et sans aspérités, mais dont il ne peut que constater la vacuité. Réel et théâtral, en ce cas, sont les deux faces d'une même difficulté à vivre: «Le réel évanescent, écrit Kattan, n'est saisi que dans la mesure où il se transforme en théâtre.» (392) Or, se transformer en théâtre engage la médiation du langage et une communication vouée à l'échec.

La plupart des pièces de Naïm Kattan reposent sur quelques canevas itératifs, où les relations se vivent par identités factices interposées et où, par conséquent, le réel nous échappe. Souvent, les personnages se rencontrent par hasard, les relations amoureuses se nouent dans un sas spatio-temporel qui n'engage ni passé ni avenir. L'identité même des personnages reste incertaine. Telle est la situation initiale de ces autres pièces que sont La discrétion, La neige, Le trajet, Le vol, Les confidences du chercheur, Sacré gamine ou La salle d'attente. Ces lieux libèrent l'imaginaire et la parole, autorisant les revirements et l'exploration des possibles. Dans d'autres pièces, l'action se situe bien après le drame, qu'il faut comprendre à rebours. Il s'agit alors de combler la distance entre des personnages qui se sont perdus de vue. C'est le cas de Les héritiers, de Ils persistent et de La convocation.

Une troisième série de pièces soulève certaines questions sociopolitiques liées à la rencontre des civilisations. Ainsi, Le seuil de la promesse, la pièce qui ouvre le volume, réunit un groupe d'immigrants de provenances diverses en attente du visa qui leur permettra de vivre au Canada. L'action se passe dans un lieu neutre, et chaque personnage porte son histoire propre qui s'exprime dans la rencontre avec les autres qui partagent le même sort. Toutefois, les amitiés nouées dans l'attente seront parfois brisées par la décision administrative qui accorde le visa souhaité aux uns et le refuse aux autres. Deux autres pièces portent sur Israël, mais en proposant une réflexion de biais. Ainsi Jacob et Esaü revient à la tradition biblique pour raconter l'aventure des deux frères et rétablir la filiation unissant juifs et musulmans. Dans Avant la cérémonie, le père est revenu assister au mariage de sa fille, qu'il n'avait pas revue depuis vingt ans: «Il a fallu l'arracher à l'ombre, le sortir de sa cachette» (69), explique Benjamin, le fiancé. C'est que David a quitté Montréal, entraînant sa famille, pour vivre en Israël. Sa femme l'a d'abord suivi, puis elle est revenue avec leur fille. Il y aura affrontement - nous sommes dans une dramaturgie du réel -, mais pas de jugement.

L'organisation du volume n'est pas chronologique, apparemment selon le vœu de l'auteur. La première partie, «Jeux de scène», réunit les pièces récentes destinées à la scène. Celles-ci sont généralement plus consistantes et proposent une action dramatique plus complexe, divisée en plusieurs scènes, voire en actes. La deuxième partie, «Jeux de paroles», réunit les pièces radiophoniques, plus brèves, dont l'action est souvent suspendue ou rapidement campée plutôt que développée, formant un 
théâtre de l'intimité, mû par la voix et la parole plutôt que par l'espace. À l'intérieur des parties, l'ordre des pièces est thématique, au détriment d'une chronologie qui aurait sans doute mis en valeur l'évolution de la pensée de Kattan. Car les pièces les plus anciennes sont les plus formalistes. Elles dissocient aisément le réel et le thêâtral dans un langage dont la logique tourne parfois à vide. Les rapports entre les personnages y sont aussi davantage stéréotypés, bien que les pièces explorent avec un certain bonheur les conséquences de la contre-culture et de ce que Jean-Marc Piotte a appelé La révolution des moeurs ${ }^{5}$. Les pièces plus récentes soulèvent plus volontiers les questions de l'héritage, jetant vers l'arrière, vers le passé, un regard qui, bien que dépourvu de nostalgie, tente le bilan, ou encore les questions liées à l'identité sociopolitique contemporaine.

L'œuvre dramatique d'Anne Hébert reste somme toute méconnue, malgré les efforts consentis par quelques spécialistes qui y recherchent, le plus souvent, les traces de la cohérence de l'œuvre entière - permutation de scènes, permanence de thèmes - , sans vraiment réfléchir à la fonction qu'exerce l'écriture dramatique dans cette œuvre pourtant monumentale. Or, écrit l'auteure, que citent les éditrices de ses Euvres complètes $^{6}$, «les premières choses que j'ai écrites, ce sont des pièces de thêâtre [...]. $\mathrm{J}^{\prime}$ en avais assez pour faire un recueil quand j'avais à peu près seize ans» (15). Ces œuvres d'adolescente ne nous sont guère parvenues, mises à part celles que Maurice Hébert avait fait éditer dans les périodiques où il écrivait lui-même: Enfants à la fenêtre, dans Le Canada français en 1938, et La boutique de Monsieur Grinsec, féerie de Noël en un acte et trois tableaux, dans L'action catholique en 1941. S'il n'y a guère de raisons de regretter la disparition de ce corpus, peut-être faut-il prendre acte néanmoins du rôle stratégique qu'a joué l'écriture dramatique dans la formation de celle qui allait devenir l'une des écrivaines les plus importantes de la francophonie. C'est en tout cas ce que permet de saisir le travail réalisé par Patricia Godbout, Annie Tanguay et Nathalie Watteyne, les deux premières surtout, dans l'édition du corpus dramatique. Car, si Anne Hébert abandonne assez rapidement les accents dickensiens qui traversent ses contes et féeries de Noël, elle conservera toujours, en la développant, cette tension déjà palpable dans la construction du personnage féminin, installé au centre de la fiction, mais doté d'une agentivité problématique.

Outre les deux déjã mentionnées, cinq pièces d'Anne Hébert avaient été publiées à ce jour: Les invités au procès, La mercière assassinée et Le temps sauvage en 1967, puis Lî́le de la demoiselle et La cage en 1990. Une autre pièce, Arche de midi, datée ici de 1944-1946, était inédite, mais assez connue des spécialistes qui en avaient consulté le manuscrit, facilement accessible dans les centres de recherche. La publication de l'œuvre dramatique intégrale ajoute à ces textes déjà connus (y

5 Jean-Marc Piotte, La révolution des mœurs. Comment les baby-boomers ont changé le Québec, Montréal, Québec Amérique, coll. «Dossiers et documents», 2016, 116 p.

6 Anne Hébert, Euvres complètes d'Anne Hébert, t. V: Théâtre, nouvelles et proses diverses, Montréal, Presses de l'Université de Montréal, coll. «Bibliothèque du Nouveau Monde», 2015, 1033 p. 
compris Arche de midi) une pièce, Adélaïde, dont le manuscrit est daté de 1978, et un livret d'opéra pour enfants daté de 1998, L'arche de Noé. L'écriture pour les jeunes publics, qui inaugure l'œuvre de l'écrivaine, n'est donc peut-être pas si marginale, après tout. L'édition critique de l'œuvre dramatique ne révèle pas de chef-d'œuvre méconnu et, il faut bien l'avouer, le discours d'escorte (introductions, commentaires critiques, bibliographies) non plus. Néanmoins, comme toute édition critique, qui oblige à lire ou à relire une œuvre dans sa version intégrale, celle-ci crée des effets de prisme qui confirment, voire accentuent les analyses antérieures tout en ouvrant des pistes nouvelles.

Ainsi, on distingue dans l'œuvre dramatique un premier cycle de pièces d'inspiration claudélienne. La rédaction de Arche du midi, entre 1944 et 1946, est parallèle à l'écriture du Torrent et contemporaine d'un article que Hébert a publié dans la Revue dominicaine en 1945 sur L'annonce faite à Marie, pièce à laquelle elle emprunte une partie de son propos quand la mort de Marie, enfant illégitime retenue sous le joug de sa mère Élisabeth, ancienne fille publique, transforme les autres personnages et leur offre une forme de rédemption. La pièce ne peut toutefois pas être ramenée à cette seule référence claudélienne puisqu'elle propose et développe cette structure de l'enfermement, liée au joug maternel, qui atteindra aussi François dans Le torrent. Campée dans un décor imprécis, «une campagne nordique», se déroulant à la fin de l'hiver sur trois journées, dont la dernière est une journée ensoleillée de printemps, la pièce est peu ancrée dans l'espace et elle se lit davantage comme un poème dramatique réparti en un chœur de voix diverses. De ce point de vue, en effet, l'écriture de Arche de midi appartient au même registre que celle des Invités au procès, texte radiophonique créé en juillet 1952 à la radio de Radio-Canada, dans le cadre de l'émission Le théâtre du Grand Prix, dans une réalisation de Guy Beaulne. "Conte radiophonique» serait un sous-titre plus approprié pour la désigner que celui de «poème» normalement accolé à la pièce, car Hébert recourt à une sorte de merveilleux médiéval en déployant ce pèlerinage de maudits (les invités) venus accomplir le procès du père Salin, dont la femme avait disparu dix années plus tôt. Un narrateur guide l'auditeur (et le lecteur) dans la sinistre auberge, où s'est arrêté un mystérieux voyageur, chargé de rétablir l'unité de l'humanité, une fois sa rédemption obtenue par le quasi-sacrifice de Ba, la fille laide, sauvée au dernier moment par l'arrivée de son chevalier.

Plus proche des Chambres de bois, autant par la date de son écriture ${ }^{7}$ que par certains motifs, notamment celui du travestissement, La mercière assassinée conserve encore quelques accents claudéliens, mais est plus proche des tonalités de L'échange que de celles de L'annonce faite à Marie. Divisée en quatre épisodes, cette dramatique destinée à la télévision est présentée à Radio-Canada en août 1958, dans une réalisation de Jean Faucher. Elle met en scène un journaliste québécois qui arrive dans un village français le jour même de l'assassinat de la mercière et qui entreprend l'enquête. Il découvre un univers aristocratique "vieille France déchue», qui écrase les jeunes femmes, travailleuses modestes, mais modernes, et qui montre combien les classes sociales sont, comme le genre sexué, d'abord un effet de performance.

7 La pièce parut la même année que le roman, dans les Écrits du Canada français, nº 4, 1958, p. 17-112. 
L'écriture du Temps sauvage est contemporaine elle aussi des Chambres de bois, bien que la pièce ait été créée à Québec, au Palais Montcalm, en octobre 1966, dans une mise en scène d'Albert Millaire. Outre le fait qu'il s'agisse de la première pièce de l'auteure à avoir été écrite pour la scène, Le temps sauvage met un terme à l'univers répressif d'une famille dominée par une mère autoritaire. D'une part, la pièce est centrée sur le personnage d'Agnès, présente en scène contrairement à la mercière de la pièce précédente, qui exprime sa révolte contre une société dominée par les hommes et par l'Église. D'autre part, l'autorité qu'elle détient sur la famille fait long feu: arrive le jour où les enfants grandissent et choisissent de voler de leurs propres ailes en quête d'un accomplissement de soi qui passe par le savoir, par l'art ou par l'amour. Agnès ne devient véritablement un grand personnage que parce que son univers s'est écroulé entre-temps. André Brochu avait noté déjà la place stratégique qu'occupent, dans l'œuvre d'Anne Hébert, Le temps sauvage et La mercière assassinée: "Ces pièces annoncent la grande période romanesque qui suit, où la nuit va prendre sa revanche sur le jour ${ }^{8}$.»

Dans le cycle de pièces plus tardives, «l'auteure se livre à une réécriture de l'histoire de la Nouvelle-France du point de vue féminin» (21). Il y a là quelque chose qui rappelle le projet qu'avait énoncé Laure Conan un siècle plus tôt. Trois pièces, écrites ou publiées entre 1970 et 1990, forment ainsi un bloc consacré à l'histoire et en particulier à la Nouvelle-France. Lîle de la demoiselle emprunte son anecdote à L'heptaméron de Marguerite de Navarre pour mettre en scène l'aventure de Marguerite de Nontron, abandonnée par Roberval sur une île du golfe du SaintLaurent. La cage propose une relecture de l'histoire de la Corriveau, présentée en contrepoint de celle de Rosalinde, épouse de John Crebessa, celui-là même qui a prononcé le jugement contre la première. Je n'insisterai pas sur ces deux pièces, ayant déjà eu l'occasion de les commenter au moment de leur parution en $1991^{9}$. Entre les deux, toutefois, l'édition critique permet de découvrir une troisième pièce jusque-là inédite et vraisemblablement inachevée, Adélaïde, dont l'action se situe à Québec vers 1860. Elle met en scène une jeune fille de bonne famille qui aspire à devenir actrice, mais qui est «envoyée chez les Ursulines par des parents qui veulent lui faire passer son goût du thêâtre» (36). Devenir actrice, Adélaïde Évanturel n'y parviendra pas puisque Anne Hébert n'a pas terminé la pièce. C'est Flora Fontanges (baptisée autrefois Marie Évanturel) qui y parviendra, et l'on rappellera l'intérêt que celle-ci portait aux figures féminines de la Nouvelle-France à travers ses pérégrinations dans la ville de Québec. On voit bien là l'intérêt que Hébert porte à la figure de l'actrice, de ces actrices qui «font partie d'un certain folklore féminin moderne» (909), mais on voit bien comment, dans ce cas particulier, l'exploration de l'agentivité féminine exige plutôt les formes narratives.

Comme le théâtre de Naïm Kattan, celui d'Anne Hébert dévoile combien la structuration du monde en classes et en genres sexués se révèle et se construit par la performance. Cependant, pour retrouver une certaine vérité, pour déployer «les

8 André Brochu, Anne Hébert. Le secret de vie et de mort, Ottawa, Presses de l'Université d'Ottawa, coll. «Evuvres et auteurs», 2000, p. 106.

9 Voir ma chronique «Ā vos classiques!» dans Voix et Images, vol. XVI, n 3, printemps 1991, p. 565-566. 
hypothèses illimitées du monde prosaïque ${ }^{10}{ }^{\prime}$, selon les mots d'Isabelle Daunais, et "concilier la représentation de l'âme avec le monde qui l'entoure ${ }^{11}$ ", selon ceux de Thomas Pavel, il vaut mieux recourir au registre narratif, qui seul peut se projeter dans le temps. Comme si le roman avait été enfoui dans le thêâtre depuis le début et qu'il n'avait suffi, somme toute, à l'auteure que de le doter d'une voix et d'une identité narrative, désormais garante du devenir. Entre le réel et le théâtral, la narration réalise la synthèse. La tension entre les genres dramatiques et les genres narratifs prend alors tout son sens, et le passage d'un registre à l'autre (et vice-versa), commun aux deux écrivains dont il est question ici, qui n'ont jamais choisi ou renoncé tout à fait, témoigne de pistes supplémentaires qu'il reste à étudier, mais que les œuvres complètes permettent d'envisager. On aurait tort cependant de conclure trop rapidement que ces œuvres dramatiques n'ont de valeur qu'en regard des romans qu'elles permettent. L'inverse est tout aussi vrai.

10 Isabelle Daunais, «Le personnage et ses qualités», Études françaises, vol. XLI, n 1, 2005, p. 16.

11 Thomas Pavel, La pensée du roman, Paris, Gallimard, coll. «NRF essais», 2003, p. 188. 